\title{
An effective energy-efficient virtual machine placement using clonal selection algorithm
}

\author{
Saiful Izwan Suliman ${ }^{1}$, Hazrien Nazman ${ }^{1}$, Afdallyna Fathiyah Harun², Roslina Mohamad", \\ Murizah Kassim $^{1}$, Farah Yasmin Abdul Rahman ${ }^{1}$ and Yuslinda Wati Mohamad Yusof ${ }^{1}$ \\ Faculty of Electrical Engineering, Universiti Teknologi MARA, 40450 Shah Alam, Selangor, Malaysia ${ }^{1}$ \\ Faculty of Computer \& Mathematical Sciences, Universiti Teknologi MARA, 40450 Shah Alam, Selangor, \\ Malaysia $^{2}$
}

Received: 14-October-2020; Revised: 11-February-2021; Accepted: 17-February-2021

(C2021 Saiful Izwan Suliman et al. This is an open access article distributed under the Creative Commons Attribution (CC BY) License, which permits unrestricted use, distribution, and reproduction in any medium, provided the original work is properly cited.

\begin{abstract}
Virtual machine (VM) placement is a process of server consolidation at the same time optimizing tasks execution in an energy-efficient environment. This process occurs in data centre which manages a number of physical machines independently. In the past decade, many research investigating virtual machine placement problem have been conducted extensively. However, most of the research focus on the energy consumptions by physical machines in a data centre. In reality, communication activities inter and intra networks is also consuming energy that should be taken into the consideration when planning for VM placement. Therefore, these two types of energy should be considered and managed in parallel during the virtual machine placement execution in order to produce energy-efficient environment. In this paper, we propose the use of Clonal Selection Algorithm (CSA) in handling virtual machine placement task which takes into consideration energy consumptions in both servers and communication network in data centre. The obtained results from the simulations produced the lowest consumption of 2219 energy unit, thus highlighting the efficiency of the proposed algorithm on the tested problem instances of different types with different complexity.
\end{abstract}

\section{Keywords}

Virtual machine placement, Clonal selection algorithm, Optimization.

\section{Introduction}

Online resource sharing has gained much attention and popularity among Internet users as this platform offers greater mobility and flexibility access. Cloud computing concept adopts this philosophy with the connected resources where they are easily accessible based on demand without direct active management by the user [1]. A central server is required to manage and disseminate resources as well as control the operations in virtual platform environment [2]. These cloud activities and operations consume a lot of energy, increasing the electricity consumption for a data centre. Based on a study conducted by Amazon, utilization of electricity in data centres accounts for more than $42 \%$ of the total operating cost, thus contributing the biggest chunk of the operation expenditure.

\footnotetext{
*Author for correspondence
}

Besides the cost factor, the generation of electricity which is mostly done using conventional method increases carbon dioxide emissions.

Therefore, it is desirable and necessary to find ways to minimize the consumption of energy, and in our case for data centre operation.

The history of cloud computing started back in 1996 by Compaq which was implemented for internal document management. This concept has since expanded as it gives many benefits to organizations especially in terms of cost and space. Cloud computing works in an environment where all available resources will be shared among authorised users to accomplish specific activities. This will reduce the need for an organization to provide multiple IT infrastructures (i.e. physical servers in many locations). Other than that, the practicality of this concept allows interactions between personnel and units running faster as resources management 
conducted with less bureaucracy which is always deemed as one of the barriers for effective implementations. Cloud computing environment also allows IT team to be prudent and proactive to accommodate and adjust to the ever fluctuating and volatile demands [3].

Server consolidation refers to the process of optimizing the server resources in an efficient way. The objective is to minimize the number of physical machines (PM) involved in an organization. This practice is essential as it can avoid the situation of under-utilized servers from happening that can lead to unnecessary use of space and resources. One of the methods for server consolidation is by implementing good exercise of virtual machine placement. This process will also consider energy-efficiency factors. In the past, various approaches for handling virtual machine placement task have been investigated. However, these studies did not consider energy consumption for communication network in data centre. However, as energy consumption by physical machine and communication network are equally significant, it is important to consider both activities in producing energy-efficient virtual machine placement.

A physical server is a computer (hardware) that provides services to other computers by processing requests and delivering information over a network. The components of a physical server such as capacity, processor, network connection, storage, and an operating system (OS) [4] are not shared across the network. A virtual machine (VM) on the other hand is a software computer utilized to imitate the process of an actual physical computer [5].

In this study, virtual servers which run on a physical machine were investigated. In this scenario, the resources of the physical machine are virtualized and distributed among all virtual machines. Even though the aim is to replace the use of physical server, the architecture of a virtual server is slightly different and more complex as it requires additional software to emulate the actual tasks.

In this paper, we propose the use of Clonal Selection algorithm (CSA) as a method to perform virtual machine placement task which considers the energy consumption in both physical servers as well as within the telecommunication network in a data centre. The main objective is to find the minimum energy consumption to perform virtual machine operation within the set constraints.

\section{Literature review}

Pires and Barán [2] reviewed a virtual machine placement taxonomy in their work. Four main techniques were discussed which are deterministic algorithm, heuristics, meta-heuristic and approximation algorithm. Five different objective functions were studied with energy consumption minimization is the most utilized $(51.2 \%)$. In terms of efficiency, meta-heuristics dominate the best performance for all objective functions.

A dynamic VM assignment and migration process in data centres was investigated by Duong-Ba et al. [1]. The focus of the study is to minimize the usage of resources as well as the power consumption by using Multi-level Join VM Placement and Migration (MJPM) algorithm. The proposed algorithm was tested on different size of problem with varied numbers of servers and hardware setting. The proposed method is effective and proven to be efficient in reducing data centre energy consumption. Choudhary et al. [4] conducted an analysis of energy consumption on virtual machine assignment methods in data centre. Eleven past research on VM placement utilizing different heuristic approached were discussed and compared. Live migration approach was found to be more effective to perform VM placement task. Therefore, the authors propose the combination of live migration utilizing dynamic threshold values to avoid stalemate process on multidimensional platform. This method will include the available resources, thus minimizing the utilization of energy.

Resource management is among the important aspect in performing efficient VM placement. Shaw et al. (2019) propose Predictive Anti-Correlated Placement Algorithm (PACPA) to predict the use of CPU and bandwidth resource consumption [5]. The existing prediction models were studied to identify improvements that can be made on the proposed algorithm. Based on the results obtained from the simulations conducted, the proposed method manages to reduce consumption energy by $18 \%$. This promising method has also reduced the service violation by over $34 \%$ compared to other algorithms. Zhao et al. [6] propose ant colony optimization algorithm (ACO) for power efficient VM assignment in cloud. In cloud computing, VM performance degradation could happen due to resource contention. Therefore, the authors have recommended the need to balance between saving physical machine power and guaranteeing virtual machine performance. The relationship between energy consumption and CPU 
utilization was first investigated and the VM degradation trend was analysed. The obtained results proof that the proposed technique manages to reduce physical machine power consumption and improve virtual machine performance.

Silva et al. [7] discussed techniques for optimizing VM assignment and migration in cloud operation. This optimization process was done by hosting several VM on each PM from different user to enable resource sharing. This allows the clients to use vast operation resources upon request, based on hosted services and users requirement. The use of the resources is controlled for effective resource management as well as to avoid non-necessary usage. Therefore, VM assignment and PM relocation are necessary to achieve the desired objectives. The authors have also highlighted the difficulties to find novel solutions and provided various problems, showing the significance of the issue in the cloud computing environment.

Liu et al. [8] proposed ant colony optimization method for VM assignment in data centre [8]. The method termed as order exchange and migration ant colony system (OEMACS). It is a hybridization between order exchange and migration (OEM) method and ant colony system (ACS) algorithm. OEMACS utilizes minimal servers needed for the VM placement. This was executed through pheromone deposition which leads the artificial ants toward optimal solutions of VM. The results obtained show that the proposed hybrid algorithm produces better VM placement as compared to other conventional heuristic and other evolutionary-based methods. It minimizes the use of energy and more efficient in utilizing the resources.

Zhao et al. [9] propose the use of hybrid genetic algorithm and tabu search algorithm. This new method termed as GATA was utilized to optimize VM placement task whilst at the same time maximizing load balance for all resources involved. Comparative studies with other existing methods were also conducted to evaluate the performance of the proposed GATA algorithm. The results gathered show that GATA algorithm is more efficient in performing VM placement with less energy consumed in an acceptable computational time.

\section{Methods}

3.1Problem formulation

Let us define

$\mathrm{V}$ a set of virtual machines (VM)
$\mathrm{P} \quad$ a set of physical machines (PM)

$V_{i} \quad$ a VM in V

$V_{i}^{c p u}$ the CPU requirement of $V_{i}$

$V_{i}^{\text {mem }}$ the memory requirement of $V_{i}$

$p_{j} \quad$ a $\mathrm{PM}$ in $\mathrm{P}$

$p_{j}^{c p u} \quad$ the CPU requirement of $p_{j}$

$p_{j}^{\text {mem }}$ the memory requirement of $p_{j}$

$p_{j}^{W c p u}$ the CPU capacity on $p_{j}$

$p_{j}^{\text {Wmem }}$ the memory capacity on $p_{j}$

$V_{p j} \quad$ the set of VMs allocated to PM

$k_{j} \quad$ the fraction of energy consumed when $p_{j}$ is

idle

$e_{j}^{\max }$ the energy usage of physical server $p_{j}$ when it is fully used

According to [10], the operation rate of the CPU in physical server $p_{j}$ can presented as

$\mu_{j}=p_{j}^{W c p u} / p_{j}^{c p u}$

Referring to the server energy utilization model, the usage of energy in physical server $p_{j}$ when its CPU utilization is $\mu_{j}$ is

$E\left(p_{j}\right)=k_{j} \cdot e_{j}^{\max }+\left(1-k_{j}\right) \cdot e_{j}^{\max } \cdot \mu_{j}$

The communication network framework of a data centre consists of three levels as depicted in Figure 1. All the virtual machines in data centre can interact with each other by using available network equipment such as a switch. This interaction consumes energy is usually overlooked as it may not consume as much energy as the server itself. However, it is important to monitor the communication energy involved in order to produce an efficient virtual machine (VM) placement. For the calculation of this communication energy utilization, four types of interactions were considered. The first type is the communication between virtual machines located on the same physical machine (PM). This interaction can be observed between vm1 and vm2 as shown in Figure 1. The second level is the interaction between virtual machines on two different physical machines, but both VMs belong to the same edge. The example of this interaction is between vm2 and vm3. The third communication type is between VMs which is located on different physical machines and different edge but in the same aggregation. This interaction could be observed between vm 3 and vm4 as shown in Figure 1. Meanwhile, the last type of communication considered in this study is between VMs on different physical machines, different edge and different aggregation. The interaction between 
vm1 and vm5 is the example of this type of communication.

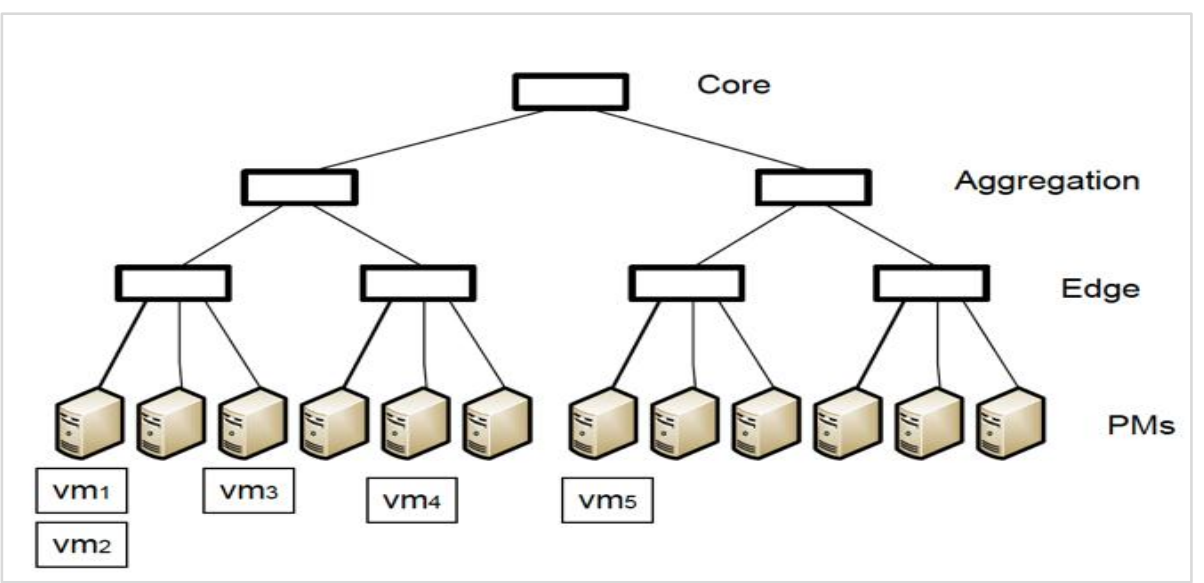

Figure 1 The communication network of a data centre

These four types of communication utilize different number of communication device(s) and different level of energy. However, for the first one where both virtual machines are on the same physical machine, no communication device is needed. Therefore, no communication energy incurred. For the second type of interaction, it utilizes one network device which is translated into one unit of energy. The third communication type needs three communication devices to execute. Therefore, it requires three unit of communication energy. For the fourth type of communication, five devices are needed to accomplish the process which translated into five unit of energy.

Let $C_{1}, C_{2}, C_{3}$, and $C_{4}$ be the four types of communication between pairs of VMs,

$C=C_{1} \cup C_{2} \cup C_{3} \cup C_{4}$

For each interaction $c \in C$, the utilization of power for transmitting a unit of data is

$e(c)=\left\{\begin{array}{l}0, \text { if } c \in C_{1} \\ e_{2}, \text { if } c \in C_{2} \\ e_{3}, \text { if } c \in C_{3} \\ e_{4}, \text { if } c \in C_{4}\end{array}\right.$

Let $E(c)$ be the energy consumption for transferring data and $l(c)$ is the amount of data involved. Therefore,

$E(c)=e(c) * l(c)$
This VM task is basically the process of assigning each virtual machine in $V$ to a physical machine in $P$. Therefore, the total energy consumption for performing this task can be calculated by adding the energy utilization to operate a physical server and the communication energy between all devices involved.

Therefore,

$F=\sum_{p_{j} \in P} E\left(p_{j}\right)+\sum_{c \in C} E(c)$

Equation (6) was utilized in this study to find the fitness value of each $\mathrm{Ab}$. It is minimized, subject to

$\cup_{p_{j} \in P} V_{p_{j}}=V$

$V_{P i} \bigcap_{p_{i} \neq p_{j}} V_{p_{j}}=\varnothing$

$p_{j}^{w_{c p u}}=\sum_{v_{i} \in V_{p_{j}}} v_{i}^{c p u} \leq p_{j}^{c p u}$

$p_{j}^{w_{m e m}}=\sum_{v_{i} \in V_{p_{j}}} v_{i}^{\text {mem }} \leq p_{j}^{\text {mem }}$

Equations (7) and (8) are necessary to ensure each $\mathrm{VM}$ is assigned to only one PM. Meanwhile equations (9) and (10) will ensure that the CPU workload and memory of the PM will not exceed its limit.

\subsection{Clonal selection algorithm}

In artificial intelligence, clonal selection algorithm is one of methods in Artificial Immune Systems (AIS). It is a metaheuristic method inspired by the fundamentals and processes of the human immune system [11]. The algorithm is modelled based on the immune system's properties of learning and memory. Immune system is the defence mechanism which 
protects our body from any foreign cell that can affect the wellbeing of our body. The utilization of AIS is based on the nature fundamental of the immune system and model its processes towards solving complex problems in many fields.

AIS algorithm is adaptive which imitates the immune processes. When an antigen (foreign cell) enters our body, the immune system will try to identify its pattern based on past encounter (if any). If the antigen has been recognized before, the immune system will use the same antibody utilised during the previous encounter to neutralize the antigen. At the same time, another process takes place to improve the performance of selected antibodies towards the antigen to increase its efficiency using evolutionary process. These processes repeated over times as antigens can mutate to become new improved cells. All these steps which occur inside our body are replicated in AIS algorithm. This method has been proven in many cases as a powerful tool to solve complex optimization dan recognition problems [12].

The standard techniques in AIS were developed based on immunological theories and process that describes the role and behaviour of the adaptive immune system. The techniques are Clonal Selection Algorithm (CSA), Negative Selection Algorithm (NSA), Immune Network Algorithms (INA), and Dendritic Cell Algorithms (DCA). In this study, the concept of clonal selection algorithm (CSA) was utilized as its algorithm as it is the most suitable and effective in solving optimization task [13].

For a population-based algorithm like CSA, a set of candidate solutions will be generated initially. These candidate solutions known as antibodies $(\mathrm{Ab})$ will be manipulated iteratively to produce desired solutions. The quality of each $\mathrm{Ab}$ is determined by using specific objective function set for each problem.

Five main steps of clonal selection algorithm were executed in this study which are initialization, affinity measure, cloning, affinity maturation (mutation) and recombination as shown in Figure 2. The determination of affinity value is very crucial as it will identify the overall quality of the generated solutions. Therefore, choosing the correct and suitable formula of mathematical equation for the objective function is very important.

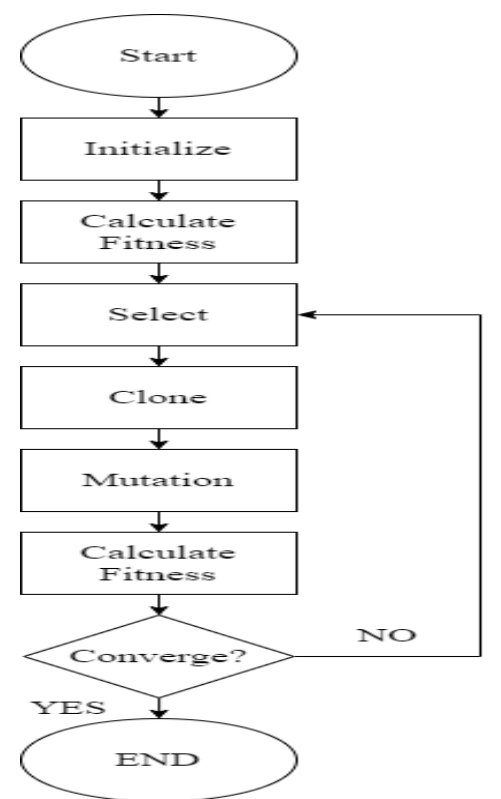

Figure 2 Clonal selection algorithm flowchart

\subsection{Generate random initial population}

Like other population-based methods, CSA is a nondeterministic algorithm which depends on the randomness on the initial candidate solutions [14]. This concept will affect the quality of the final solutions as manipulations process through genetic operators are conducted on the initial population. In the case of this study, if the generated initial Ab's are of high quality, then optimal solutions could be possibly produced. Two variables were involved in the generation of the initial population, which are virtual machine (VM) and physical machine (PM). A $\mathrm{PM}$ is the real machine utilized in a network, whereas a VM is a software that imitates the functions of a PM [15]. A physical machine can be a host ta a number of virtual machines. In this study, a total of 50 random antibodies were generated for the initial population of candidate solutions. Table 1 shows the example of initial population of antibodies that were generated randomly. The number of PM and VM utilized is dependent on the size of the problem. For example, if we try to assign fifty (50) VMs on twenty (20) PMs, then the task will consist of PM1 to PM20 and VM1 to VM50. Each Ab will have fifty genes (properties) that represent the assignment of which PM to each VM. Due to the randomness element of the gene generation, each $\mathrm{Ab}$ will have a set of different genes from other $\mathrm{Ab}$. 
The size of the population affects the performance of the algorithm in two aspects which are the quality of the produced solutions and the computational time taken to produce the final solutions. Based on previous studies, population with the size of fifty is ideal to produce the desired solutions within acceptable computational time [16].

Table 1 Example of an initial population of antibodies

\begin{tabular}{|c|c|c|c|c|c|}
\hline & $\mathrm{VM}_{1}$ & $\mathrm{VM}_{2}$ & $\mathrm{VM}_{3}$ & $\mathrm{VM}_{(\mathrm{t}-1)}$ & $\mathrm{VM}_{\mathrm{t}}$ \\
\hline$A b_{1}$ & $\mathrm{PM}_{1}$ & $\mathrm{PM}_{13}$ & $\mathrm{PM}_{19}$ & $\mathrm{PM}_{7}$ & $\mathrm{PM}_{11}$ \\
\hline$A b_{2}$ & $\mathrm{PM}_{2}$ & $\mathrm{PM}_{1}$ & $\mathrm{PM}_{3}$ & $\mathrm{PM}_{9}$ & $\mathrm{PM}_{8}$ \\
\hline$A b_{3}$ & $\mathrm{PM}_{9}$ & $\mathrm{PM}_{2}$ & $\mathrm{PM}_{1}$ & $\mathrm{PM}_{15}$ & $\mathrm{PM}_{17}$ \\
\hline \multicolumn{6}{|l|}{..... } \\
\hline$A b_{(t-1)}$ & $\mathrm{PM}_{1}$ & $\mathrm{PM}_{18}$ & $\mathrm{PM}_{14}$ & $\mathrm{PM}_{19}$ & $\mathrm{PM}_{18}$ \\
\hline$A b_{t}$ & $\mathrm{PM}_{8}$ & $\mathrm{PM}_{10}$ & $\mathrm{PM}_{17}$ & $\mathrm{PM}_{1}$ & $\mathrm{PM}_{2}$ \\
\hline
\end{tabular}

\subsubsection{Fitness value calculation}

The quality of each $\mathrm{Ab}$ in clonal selection algorithm depends on the fitness value towards the objective function. Equation (6) was utilized for this purpose. It will calculate the total energy consume to complete the VM placement task. This objective function is the standard equation that utilized by many researchers involved in this area [17]. As the main objective of this study is to minimize the energy consumption, therefore antibody with the lowest fitness value is considered the best solution.

3.3.2Sorting and selection

After the affinity measurement process takes place, the entire population of antibodies will be sorted based on the value of its fitness value. As this study concerns with minimizing the energy consumption, the antibodies will be sorted in ascending order. It means that the first antibody will have the lowest fitness value (energy consumption). From this sorted population, the top $\mathrm{m}$ antibodies were selected for the next phase which is the cloning process. The value of parameter $m$ was investigated in the initial study and it was found that five (5) antibodies the optimal value in order to produce good solution within an acceptable computational time. Table 2 shows the example of five chosen sorted antibodies produced from this process.

Table 2 Sorted antibodies

\begin{tabular}{|c|c|c|c|c|c|}
\hline $\mathbf{A b}$ & Genes & & & & Cost \\
\hline$A b_{1}$ & $\mathrm{PM}_{7}$ & $\mathrm{PM}_{1}$ & $\mathrm{PM}_{19}$ & $\mathrm{PM}_{20}$ & 2024 \\
\hline$A b_{2}$ & $\mathrm{PM}_{5}$ & $\mathrm{PM}_{4}$ & $\mathrm{PM}_{1}$ & $\mathrm{PM}_{16}$ & 2048 \\
\hline$A b_{3}$ & $\mathrm{PM}_{1}$ & $\mathrm{PM}_{5}$ & $\mathrm{PM}_{20}$ & $\mathrm{PM}_{8}$ & 2256 \\
\hline$A b_{4}$ & $\mathrm{PM}_{3}$ & $\mathrm{PM}_{2}$ & $\mathrm{PM}_{10}$ & $\mathrm{PM}_{17}$ & 2478 \\
\hline$A b_{5}$ & $\mathrm{PM}_{1}$ & $\mathrm{PM}_{2}$ & $\mathrm{PM}_{10}$ & $\mathrm{PM}_{20}$ & 2689 \\
\hline
\end{tabular}

\subsection{Cloning}

In this process, antibodies with low fitness values selected from the previous stage were multiplied (duplicated) several times. The selected antibodies are the best candidate solutions produced at that stage. Therefore, multiplying its numbers will increase the chance of producing better antibodies during the next process [18]. In this study, each selected antibody was cloned four times. Therefore, from the selected five antibodies, a pool of twenty cloned antibodies were produced. Table 3 shows the example of $\mathrm{t}$ cloned antibodies with each selected antibody cloned once.

Table 3 Cloned antibodies

\begin{tabular}{llllll}
\hline$A b_{1}$ & $\mathrm{PM}_{10}$ & $\mathrm{PM}_{1}$ & $\mathrm{PM}_{19}$ & $\mathrm{PM}_{20}$ & $\mathrm{PM}_{18}$ \\
\hline$A b_{2}$ & $\mathrm{PM}_{10}$ & $\mathrm{PM}_{1}$ & $\mathrm{PM}_{19}$ & $\mathrm{PM}_{20}$ & $\mathrm{PM}_{18}$ \\
\hline$A b_{3}$ & $\mathrm{PM}_{1}$ & $\mathrm{PM}_{15}$ & $\mathrm{PM}_{20}$ & $\mathrm{PM}_{8}$ & $\mathrm{PM}_{5}$ \\
\hline$A b_{4}$ & $\mathrm{PM}_{1}$ & $\mathrm{PM}_{15}$ & $\mathrm{PM}_{20}$ & $\mathrm{PM}_{8}$ & $\mathrm{PM}_{5}$ \\
\hline$A b_{5}$ & $\mathrm{PM}_{12}$ & $\mathrm{PM}_{2}$ & $\mathrm{PM}_{10}$ & $\mathrm{PM}_{20}$ & $\ldots$ \\
\hline$\ldots$ & $\ldots$. & $\ldots$ & $\ldots$ & $\ldots$ & $\ldots$ \\
\hline $\mathrm{C}_{(\mathrm{t}-1)}$ & $\mathrm{PM}_{14}$ & $\mathrm{PM}_{4}$ & $\mathrm{PM}_{1}$ & $\mathrm{PM}_{16}$ & $\mathrm{PM}_{13}$ \\
\hline $\mathrm{C}_{\mathrm{t}}$ & $\mathrm{PM}_{14}$ & $\mathrm{PM}_{4}$ & $\mathrm{PM}_{1}$ & $\mathrm{PM}_{16}$ & $\mathrm{PM}_{13}$ \\
\hline
\end{tabular}




\subsection{Mutation}

The cloned selected antibodies with good fitness value will undergo affinity maturation process. In this process, a property of the antibody (gene) will be mutated. This property represents an important information describing the uniqueness of the antibody. Modifying this gene will alter the overall description of the antibody. However, there is no guarantee that this mutation process will produce a better antibody and drive the whole algorithm towards the desired solutions. In this step, a probability value will be used to determine whether an antibody will undergo affinity maturation process or not. This probability value varies between 0.07 $(7 \%)$ to $0.11(11 \%)$. This value must not be too high so that the fundamental of an antibody remains. If the probability value is too high, this mutation process may change the antibody to an entirely new antibody. Initial study was conducted in this study, it was found that mutation probability of 0.07 produces the best result towards the objective function. After the mutation process, the fitness value of each mutated antibody will be calculated to identify its quality. Then, this pool of mutated antibodies will be sorted based on the fitness value.

\subsection{Generate new population}

A new pool of antibodies was constructed for each new iteration. This new population consists of:

i. The top ten antibodies from the population of mutated antibodies

ii. Forty antibodies are randomly generated.

These antibodies will undergo the same processes which are affinity measurement, sorting, selection, cloning and affinity maturation (mutation). These steps are repeated until the termination criterion is exhausted.

\subsection{Termination}

For solving optimization problem using metaheuristic method such as Clonal Selection Algorithm, repetition of the similar processes is crucial towards the optimal solution [19]. However, this may also lead to a local optimum that will give undesired result. Other than that, the best solution might be produced earlier than the last iteration set in the algorithm. This will only lengthen the computational time for the whole process. Therefore, it emphasizes the need to have a good termination criterion for the algorithm. For this initial study, iteration limit of 1000 was utilized, and the best result produced was taken as the optimal solution.
Clonal selection algorithm is a population-based metaheuristic approach. The first step is the generation of initial set of candidate solutions through randomness elements. Due to this concept, in order to identify the best solution, it is impossible to be achieved in just a single run of simulation. It requires many times of simulation executions before the best solution is identified.

The proposed CSA for performing virtual machine placement task was executed on Window-based operating system computer with specification of i7 Pentium processor and $8 \mathrm{~GB}$ RAM. The storage capacity of the computer is $512 \mathrm{~GB}$. The results obtained from the study is discussed in the next section.

\section{Result and analysis}

As mentioned in the previous section, five probability of mutation values ranging from 0.07 to 0.11 were investigated to be incorporated into the clonal selection algorithm. The value of mutation probability is very crucial as it can determine the quality of the solutions produced. Based on the initial investigation conducted on this matter, the results were very conclusive as presented in Table 4.

From the five values tested, clonal selection algorithm performs the best at 0.07 value for probability of mutation. The energy consumption for the virtual machine placement was minimized to 2216.8 which is the lowest among all five values. For the remaining four probability values, the energy consumptions produced by the algorithm vary from 2514 to 3262 with incorporation of 0.08 into the algorithm is the worst performer.

The analysis of these results is illustrated in a line graph as shown in Figure 3. The fluctuation line represents the inconsistency between the result produced by one probability value to another. This is most probably due to the element of randomness utilized in the proposed method. However, despite this element, clonal selection algorithm utilizing 0.07 as the probability of mutation outperforms other values in minimizing the energy consumption for VM placement task.

Figure 4 shows the pattern of energy consumption when solving VM placement task using clonal selection algorithm in 1000 iterations. As can be seen from the graph, the proposed method has significantly minimized the energy consumption for the task. During the first iteration, the algorithm 
generated 2920 unit of energy consumption. This consumption has been reduced gradually until the last iteration which produces 2119 unit of energy. This represents $27.43 \%$ reduction of utilized energy.

Analysis conducted on the result shows that the lowest value of 2119 was achieved during the $940^{\text {th }}$ iteration. It remains at this value until the algorithm stops at the iteration limit of 1000 . The proposed method could not further decrease the energy consumption after the $940^{\text {th }}$ iteration as it has probably reached the optimal solution.

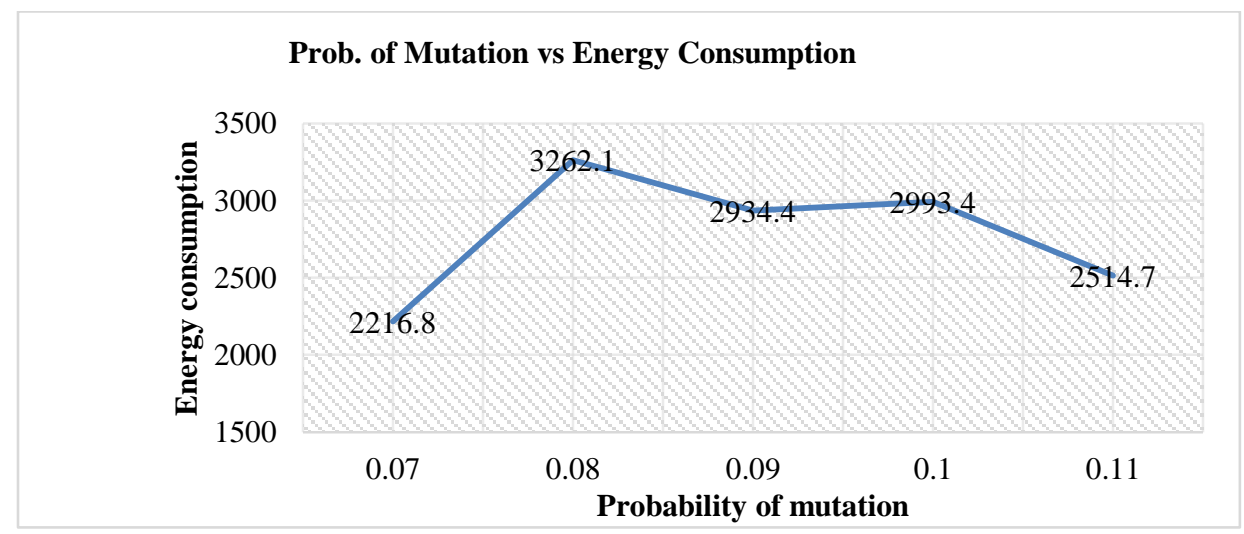

Figure 3 Energy consumption for each of probability of mutation

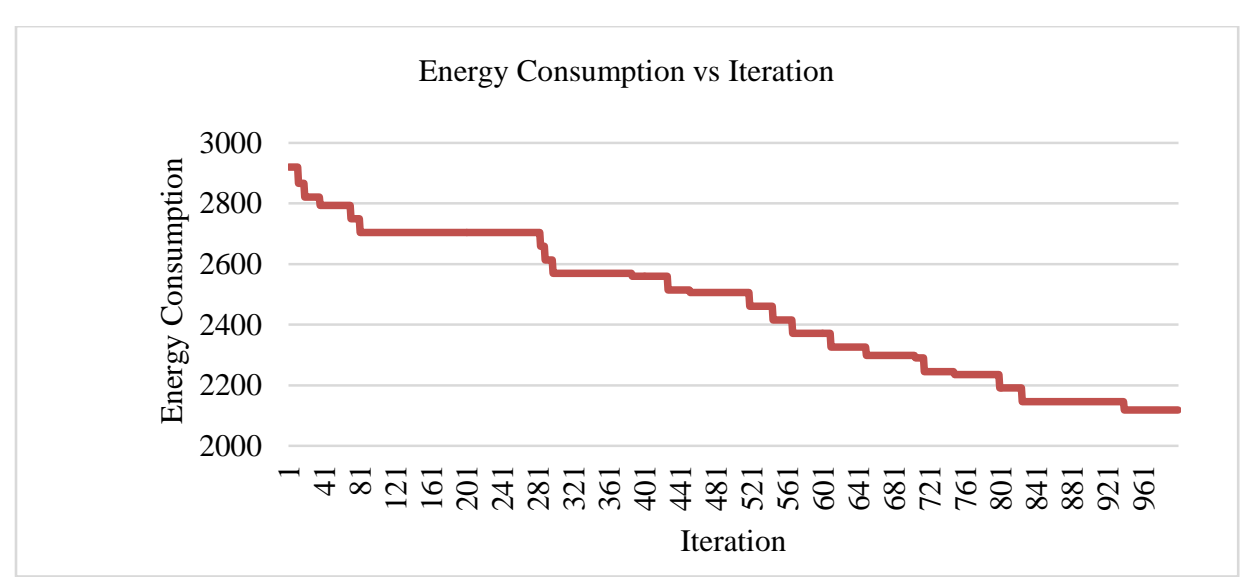

Figure 4 Energy consumption in 1000 iterations

\section{Conclusion}

Clonal selection algorithm has been successfully investigated and implemented in this study to perform virtual machine placement task. In most of the previous studies conducted on the same problem domain, only the energy consumed by the physical machine was investigated. However, that is not the case for real VM placement task. The energy needed for communications between the machines involved in this task must also be considered. This scenario was investigated by using the proposed algorithm with different parameter settings. The obtained results show the capability of clonal selection algorithm in producing good virtual machine placement with low energy consumption. This highlights the suitability of the proposed method in solving optimization problem such as VM placement. In order to analyze the efficiency of clonal selection algorithm in future work, further study should be conducted to compare its performance against other existing methods in performing VM placement task. Besides that, the parameter settings of the algorithm can also be further investigated especially the size of the population number of clones as well as the stopping criterion. Hybridization of CSA with other methods could also be looked into in order to improve its performance in optimization-related problem in general. The proposed method shows promising performance that can be further improved with detailed studies in the future. 


\section{Acknowledgment}

The authors would like to express the gratitude to the Ministry of Higher Education, Malaysia and Universiti Teknologi MARA, Selangor, Malaysia for the financial support given for this project [FRGS Grant 600-RMI/FRGS 5/3 (0007/2016)].

\section{Conflicts of interest}

The authors have no conflicts of interest to declare.

\section{References}

[1] Duong-Ba TH, Nguyen T, Bose B, Tran TT. A dynamic virtual machine placement and migration scheme for data centers. IEEE Transactions on Services Computing. 2018:1-11.

[2] Pires FL, Barán B. A Virtual machine placement taxonomy. In 15th IEEE/ACM international symposium on cluster, cloud and grid computing 2015 (pp. 159-68). IEEE.

[3] Jonas E, Schleier-Smith J, Sreekanti V, Tsai CC, Khandelwal $\mathrm{A}, \mathrm{Pu} \mathrm{Q}$, et al. Cloud programming simplified: a berkeley view on serverless computing. arXiv preprint arXiv:1902.03383. 2019.

[4] Choudhary A, Rana S, Matahai KJ. A critical analysis of energy efficient virtual machine placement techniques and its optimization in a cloud computing environment. Procedia Computer Science. 2016; 78:132-8.

[5] Shaw R, Howley E, Barrett E. An energy efficient anti-correlated virtual machine placement algorithm using resource usage predictions. Simulation Modelling Practice and Theory. 2019; 93:322-42.

[6] Zhao H, Wang J, Liu F, Wang Q, Zhang W, Zheng Q. Power-aware and performance-guaranteed virtual machine placement in the cloud. IEEE Transactions on Parallel and Distributed Systems. 2018; 29(6):1385400.

[7] Silva Filho MC, Monteiro CC, Inácio PR, Freire MM. Approaches for optimizing virtual machine placement and migration in cloud environments: a survey. Journal of Parallel and Distributed Computing. 2018; 111:222-50.

[8] Liu XF, Zhan ZH, Deng JD, Li Y, Gu T, Zhang J. An energy efficient ant colony system for virtual machine placement in cloud computing. IEEE Transactions on Evolutionary Computation. 2016; 22(1):113-28.

[9] Zhao DM, Zhou JT, Li K. An energy-aware algorithm for virtual machine placement in cloud computing. IEEE Access. 2019; 7:55659-68.

[10] Ahmad A, Zainudin WS, Kama MN, Idris NB, Saudi MM. Cloud Co-residency denial of service threat detection inspired by artificial immune system. In proceedings of the artificial intelligence and cloud computing conference 2018 (pp. 76-82).

[11] Hofmeyr SA, Forrest S. Architecture for an artificial immune system. Evolutionary Computation. 2000; 8(4):443-73.

[12] Rashid N, Iqbal J, Mahmood F, Abid A, Khan US, Tiwana MI. Artificial immune system-negative selection classification algorithm (NSCA) for four class electroencephalogram (EEG) signals. Frontiers in Human Neuroscience. 2018; 12:1-15.

[13] Luo W, Lin X, Zhu T, Xu P. A clonal selection algorithm for dynamic multimodal function optimization. Swarm and Evolutionary Computation. 2019; 50:100459.

[14] Zamani MK, Musirin I, Omar MS, Suliman SI, Ghani NA, Kamari NA. Gravitational search algorithm based technique for voltage stability improvement. Indonesian Journal of Electrical Engineering and Computer Science. 2018; 9(1):123-30.

[15] Donyagard Vahed N, Ghobaei-Arani M, Souri A. Multiobjective virtual machine placement mechanisms using nature-inspired metaheuristic algorithms in cloud environments: a comprehensive review. International Journal of Communication Systems. 2019; 32(14):1-32.

[16] Suliman SI, Kendall G, Musirin I. Artificial immune algorithm in solving the channel assignment task. In international conference on control system, computing and engineering (ICCSCE 2014) 2014 (pp. 153-8). IEEE.

[17] Parvizi E, Rezvani MH. Utilization-aware energyefficient virtual machine placement in cloud networks using NSGA-III meta-heuristic approach. Cluster Computing. 2020:1-23.

[18] Suliman SI, Rahman TA. Artificial immune system based machine learning for voltage stability prediction in power system. In international power engineering and optimization conference 2010 (pp. 53-8). IEEE.

[19] Chen D, Li S, Wang J, Feng Y, Liu Y. A multiobjective trajectory planning method based on the improved immune clonal selection algorithm. Robotics and Computer-Integrated Manufacturing. 2019; 59:431-42.

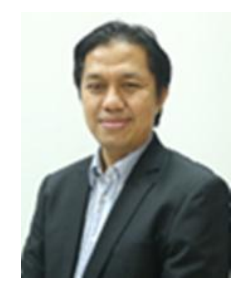

Saiful Izwan Suliman is a senior lecturer at the Faculty of Electrical Engineering, Universiti Teknologi MARA (UiTM), Malaysia. He obtained 1st class degree in Artificial Intelligence in 2002 and Master of Science (Electrical Engineering) from UiTM in 2006. His Phd was awarded by The University of Nottingham, UK in 2015. His research interest is mainly in the area or artificial intelligence, optimization and pattern recognition algorithm, metaheuristic approach, frequency bandwidth spectrum and power system operations. He has published papers in many international conferences as well as reputable journals. $\mathrm{He}$ is a certified Professional Technologist and Chartered Engineer (CEng) with IET, UK. He is currently the head of Innovation Unit, Office of Deputy Vice Chancellor (Research \& Innovation) at Universiti Teknologi MARA.

Email: saifulizwan@uitm.edu.my 


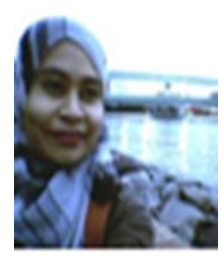

Afdallyna Fathiyah Harun earned her $\mathrm{PhD}$ from University of Nottingham, UK in 2014. While her Masters and Bachelor degree were obtained from Universiti Teknologi MARA Malaysia. Her background is mainly on Information Technology and Human Computer Interaction. She is currently a senior lecturer in Universiti Teknologi MARA Malaysia where her current research interest includes Technology Appropriation, Participatory Design and Technology Design.

Email: hazrien.nazman@gmail.com

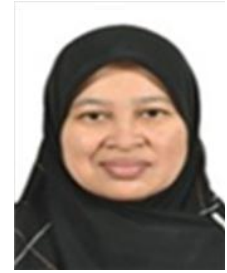

Murizah Kassim is currently working as Associate Professor at the Faculty of Electrical Engineering, Universiti Teknologi MARA, Shah Alam, Selangor. She received her Ph.D. in Electronic, Electrical, and System Engineering in 2016 from the Faculty of Built Environment and Engineering, Universiti Kebangsaan Malaysia (UKM). She has published many indexed papers related to a computer network, IoT, Web, and Mobile development applications research. She experienced for 19 years in the technical team at the Centre for Integrated Information System, UiTM. She is also head of Enabling Internet of Things Technologies (EIIoTT) research group UiTM. She joined the academic since January 2009 and currently a member of MBOT, IEEE, IET, IAENG, and IACSIT organization. Email: afdallyna@tmsk.uitm.edu.my

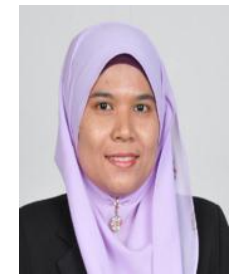

Roslina Mohamad obtained the B. Eng. degree in Electrical Engineering and M. Eng. Science degree from Universiti Malaya, Kuala Lumpur in 2003 and 2008 respectively. She received the $\mathrm{PhD}$. degree in Aerospace Engineering from Universiti Putra Malaysia, in 2016. From 2006 until now, she is with Faculty of Electrical Engineering, Universiti Teknologi MARA, as a senior lecturer. Her research interests are in Computing Algorithm and Digital Signal Processing for Deep Space Communication, Channel Coding, Theory of Computation, and Wireless Communication.

Email: roslina780@uitm.edu.my

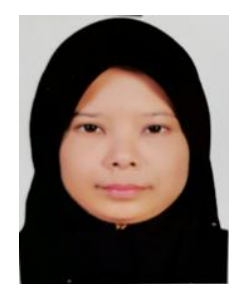

Farah Yasmin Abdul Rahman is a senior lecturer at the Faculty of Electrical Engineering, Universiti Teknologi MARA (UiTM), Malaysia. She has B. Eng (Hons.) Electrical from Universiti Teknologi MARA in 2003. Next, in 2005 she further her study in Universiti Kebangsaan Malaysia and awarded M.Eng Communication and Computer. Her research areas are Image Processing, Artificial Intelligence and Embedded System.

Email: murizah@uitm.edu.my

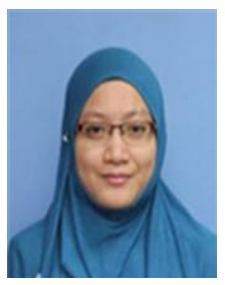

Yuslinda Wati Mohamad Yusof is a senior lecturer at the Faculty of Electrical Engineering, Universiti Teknologi MARA (UiTM), Malaysia. She obtained $1^{\text {st }}$ class degree in Electrical Engineering in 2004 and Master of Science (Electronics System Design Engineering) from USM in 2006. Currently, she is pursuing Phd at UiTM. Her research interest is mainly in the area of Image Processing, Embedded System and IoT applications.

Email: farahy@uitm.edu.my 